\title{
Study of Spoof Plasmon Formed on Periodically Corrugated Metal Surface Based on Cavity Resonance Method ${ }^{*}$
}

\author{
Kazuo OGURA, Kei SEKINE, Yuta ANNAKA and Min Thu SAN ${ }^{1)}$ \\ Graduate School of Science and Technology, Niigata University, Niigata 950-2181, Japan \\ ${ }^{1)}$ West Yangon Technological University, Yangon, Myanmar
}

(Received 26 September 2018 / Accepted 18 November 2018)

\begin{abstract}
We experimentally studied spoof plasmons developed on a metal cylinder with rectangular corrugations. Cavity resonance method using vector network analyzer is employed to examine electromagnetic properties of the spoof plasmon. The dispersion characteristics are obtained using resonance frequencies and can be recognized as two regions: bounded and hybrid surface-wave regions. The bounded surface wave is formed near the upper cutoff frequency, while the hybrid surface wave similar to a Sommerfeld wave is formed away from the upper cutoff frequency. In addition to the dispersion characteristics, the reflectance of spoof plasmon at the corrugation end is examined, based on quality factors of resonant modes.
\end{abstract}

(C) 2019 The Japan Society of Plasma Science and Nuclear Fusion Research

Keywords: periodic structure, surface wave, spoof plasmon, cavity resonance method, dispersion characteristic, quality factor, end reflectance

DOI: $10.1585 /$ pfr. 14.2406008

\section{Introduction}

Spoof plasmons are surface waves excited along metallic corrugated surfaces and are considered to be the microwave and terahertz equivalent of optical surface plasmon polaritons for metals. Metamaterials like gratings or corrugated walls have surfaces with sub-wavelength structures, which form spoof plasmons [1]. Spoof plasmons are now attracting attention in the terahertz application areas such as coherent terahertz radiation sources, plasma heating and diagnostics, and material research. And the highly confined guiding of spoof plasmon has been verified using a planar plasmonic metamaterial [2]. For a cylindrical geometry, the boundary condition on the side ends of the planar corrugation is replaced by an azimuthally periodic condition in the cylindrical coordinate system, resulting in axisymmetric and non-axisymmetric cylindrical spoof plasmons [3]. Since the cylindrical spoof plasmons cling to the corrugated cylinder and are reflected at the corrugation ends, a resonator may be formed in the same way as in a conventional waveguide cavity.

The spoof-plasmon-based surface-wave resonator enables intense generations in microwave and terahertz wave regions [4-6]. End reflections play an important role in the resonator formation. They also affect both starting conditions and saturation effects of the microwave source [7,8]. Hence, it is very important to understand such properties as the reflectance of spoof plasmons for future applications. However, it is very difficult to analyze the reflectance in real devices even using computer simulation $[9,10]$.

author'se-mail: teogura@eng.niigata-u.ac.jp

*) This article is based on the presentation at the 12th International Conference on Open Magnetic Systems for Plasma Confinement (OS2018).
In this paper, a spoof plasmon on a corrugated cylinder is studied. There is another kind of cylindrical surface wave caused by surface plasmons, i.e., Sommerfeld wave, the dispersion characteristics of which are fixed by the physical properties of the metal. On the other hand, spoof plasmons due to the periodic structure can have an arbitrary dispersion and may be very useful for generation and guiding of electromagnetic waves up to the terahertz region. In practical situations, the extent of metamaterial is finite. Reflections from ends quantize the spoof plasmon into discrete resonant modes. These discrete modes are examined by applying a cavity resonance method, in which microwave reflection, transmission, and input impedance are measured as functions of frequency by a vector network analyzer (VNA) [9-12]. The dispersion characteristics of spoof plasmons on the corrugated surface are examined by comparing the numerical and experimental frequencies. And the reflection coefficients of spoof plasmons at the corrugation end are examined using the quality factors of resonant modes.

\section{Cavity Resonance Method using VNA}

The experimental set-up is shown in Fig. 1. A VNA (Anritsu 37269D) is employed to measure the scattering parameters: the reflections from the structure to port 1 $\left(\mathrm{S}_{11}\right)$ and port $2\left(\mathrm{~S}_{22}\right)$, as well as the transmissions through the structure from port 1 to port $2\left(\mathrm{~S}_{12}\right)$ and vice versa $\left(\mathrm{S}_{21}\right)$. A needle antenna is employed to excite the spoof plasmons. However, the coupling of the needle antenna to the spoof plasmon is too weak to excite the surface-wave resonator. We use a combination of a reflector and the nee- 


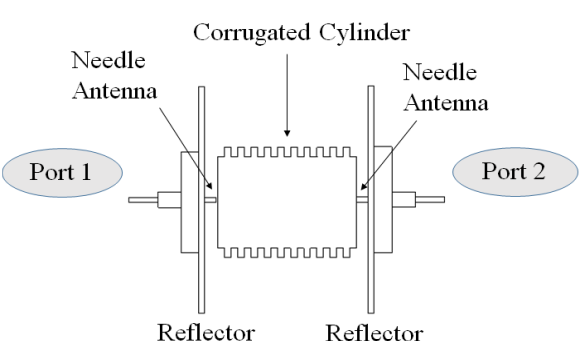

Fig. 1 Schematic of the experimental set-up for scattering parameters measurement using VNA.

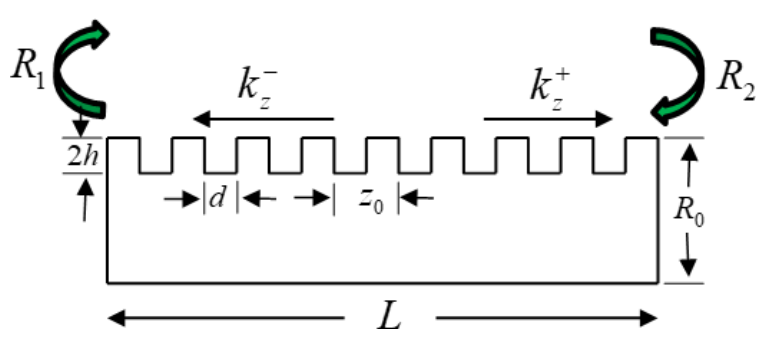

Fig. 2 Forward and backward spoof plasmons in a finite length corrugated structure are represented by their wavenumbers $k_{z}^{+}$, and $k_{z}^{-}$, respectively. The effects of reflection are taken into account using the reflection coefficients of $R_{1}$ and $R_{2}$ at each end of the structure.

dle shown in Fig. 1, where the surface-plasmon polariton is excited on the reflector surface and interacts with the spoof plasmon on the corrugation [12].

Figure 2 shows how a resonance is formed by the spoof plasmon. Forward spoof plasmon $k_{z}^{+}$coming from the left end of the structure reflects with a reflection coefficient $R_{2}$ at the structure's right end. The reflected spoof plasmon $k_{z}^{-}$propagates to the left end and reflects with a reflection coefficient $R_{1}$. Forward and backward spoof plasmons form an axil resonator like in a waveguide cavity $[9,10,13]$. The reflection coefficient for one round trip in the structure is $R=R_{1} R_{2}$.

The corrugation is made of aluminum alloy A5052 and the parameters are the average corrugation radius $R_{0}=13 \mathrm{~mm}$, corrugation amplitude $h=1 \mathrm{~mm}$, corrugation width $d=1.5 \mathrm{~mm}$ and periodic corrugation length $z_{0}=3.0 \mathrm{~mm}$. The cylinder has a number of period $N=10$, and the length of the cylinder is $L=10 z_{0}$. A closed cavity is formed when a finite-length corrugation is shorted at both ends by the reflectors like in Fig. 1. In general, the closed cavity having $N$ number of periods will support axial resonant modes at wavenumber $k_{\mathrm{z}}=\left(\pi / z_{0}\right)(n / N)$, where $n$ ranges from 0 to $N$ [9]. When one end of a corrugated resonator is left open, this cavity is referred to as an open cavity [10]. In contrast to the closed cavity, the open cavity supports axial resonant modes with "half-integer" spacing, namely at $k_{\mathrm{z}}=\left(\pi / z_{0}\right)(n+1 / 2) / N$ with $n$ ranging from 0 to $N-1$.

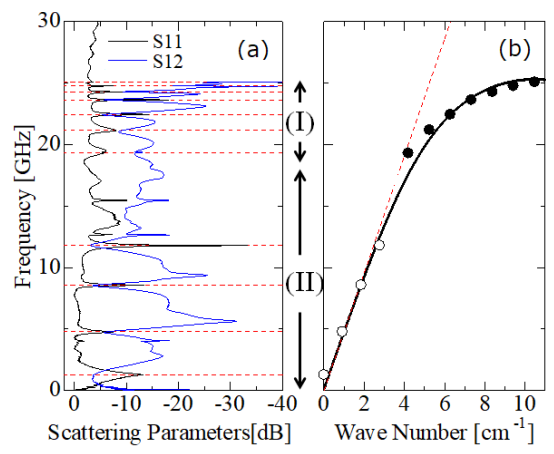

Fig. 3 (a) Reflection $\left(S_{11}\right)$ and transmission $\left(S_{12}\right)$ profiles of a corrugated cylinder for the closed cavity. Red dotted lines correspond to resonances. (b) Resonance frequencies versus wavenumber for a spoof plasmon. The dashed line represents the light line. See the text for the closed and open circles.

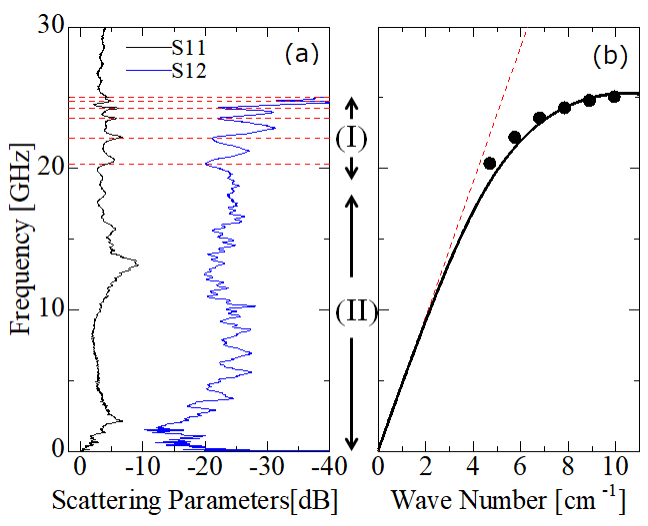

Fig. 4 (a) Reflection $\left(S_{11}\right)$ and transmission $\left(S_{12}\right)$ profiles of a corrugated cylinder for the open cavity. Red dotted lines correspond to resonances. (b) Resonance frequencies versus wavenumber for a spoof plasmon. The dashed line represents the light line. See the text for the closed circles.

The resonances appear as spikes where the reflection decreases while the transmission increases. The measured $S_{11}$ and $S_{12}$ are shown in Figs. 3 and 4. The set-up for Fig. 3 has a reflector on both sides and corresponds to the closed cavity. For Fig. 4, the reflector of the excitation antenna on port-2 side is removed with the needle antenna remaining and the set-up becomes an open cavity. Experimentally observed resonant spikes in $S_{11}$ and $S_{12}$ are depicted by circles in Figs. 3 (b) and 4 (b) for corresponding axial resonant modes. The dispersion curves of the spoof plasmon in these figures are derived numerically based on a mathematical formulation for rectangular corrugations presented in $[12,14]$.

For the closed cavity, seven resonances in the region (I) of Fig. 3 (a) are plotted by closed circles at $k_{\mathrm{z}} \mathrm{z}_{0}=$ $4 \pi / 10,5 \pi / 10,6 \pi / 10,7 \pi / 10,8 \pi / 10,9 \pi / 10$ and $10 \pi / 10$ in Fig. 3 (b). For the $10 \pi / 10$ resonance, a very weak $S_{21}$ signal under $-25 \mathrm{~dB}$ can be seen and no meaningful $S_{11}$ 
signal is observed. The spoof plasmon far from the upper cutoff frequency has a dispersion relation very close to the light line and forms hybrid surface wave as pointed out in Refs. [1, 12]. The hybrid surface wave is hard to distinguish from the "real" surface-plasmon polariton, which is the Sommerfeld wave in our cylindrical geometry. The axial resonant modes based on the Sommerfeld wave are also examined by replacing the corrugated cylinder with a straight one having the length of $30 \mathrm{~mm}$ and the diameter of $26 \mathrm{~mm}$, corresponding to the parameters of the corrugated cylinder. The resonances due to the Sommerfeld wave are almost the same as those in region (II) of Fig. 3 (a). In this case, the cavity length for resonators should be the distance $L_{\text {ref }}$ between two end reflectors of Fig. $1, L_{\text {ref }}=34 \mathrm{~mm}$ in our experiments. Their resonant wavenumbers are $m \pi / L_{\text {ref }}$ with integer $m$, plotted by open circles in Fig. 3 (a).

For the open cavity, six resonances are observed in region (I), as shown in Fig. 4 (a). In Fig. 4(b), these resonances are plotted at the corresponding wavenumber of $k_{\mathrm{z}} \mathrm{z}_{0}=\pi(4+1 / 2) / 10, \pi(5+1 / 2) / 10, \pi(6+1 / 2) / 10$, $\pi(7+1 / 2) / 10, \pi(8+1 / 2) / 10$, and $\pi(9+1 / 2) / 10$. Theoretical and experimental results agree reasonably well. Resonances in region (II) disappear in Fig. 4 (a), showing that the end reflectance of the hybrid surface wave is too weak to form resonators without reflectors.

\section{Quality Factors and Reflectance of Finite Length Corrugated Cylinder}

We examine reflectance at the corrugation end based on quality-factor measurement. There are three quality factors: the total quality factor $Q_{T}$, the ohmic quality factor $Q_{\Omega}$, and the diffractive quality factor $Q_{d}$. The ohmic wall loss in the cavity determines $Q_{\Omega}$, and $Q_{d}$ depends on the radiative power loss at the corrugation end and is closely related to the end reflectance of resonator. The three quality factors are related the relationship as $1 / Q_{T}=1 / Q_{\Omega}+1 / Q_{d}$.

The factor $Q_{T}$ can be derived based on the well-known half-power points' method as

$$
Q_{T}=\frac{f_{r}}{\Delta f}
$$

where $f_{r}$ is the resonance frequency determined at the maximum magnitude of the transition while $\Delta f$ is frequency difference between the half-power points $(1 / \sqrt{2}$ points of the maximum transmission magnitude). The Smith chart is plotted in Fig. 5, where three loops for axial resonant modes of $7 \pi / 10,8 \pi / 10$, and $9 \pi / 10$ are presented. Couplings between the resonator and the excitation antenna are under-couplings.

The factor $Q_{\Omega}$ may be derived using the critical-points method [11]. This method uses input-impedance $Z_{i}$ and is useful even for our weak under-coupling cases. In Fig. 6, two critical-points are defined as the points at which the corresponding reactance is maximal $\left(f_{1}\right)$ and minimal $\left(f_{2}\right)$; another two frequencies $f_{3}$ and $f_{4}$ are defined at

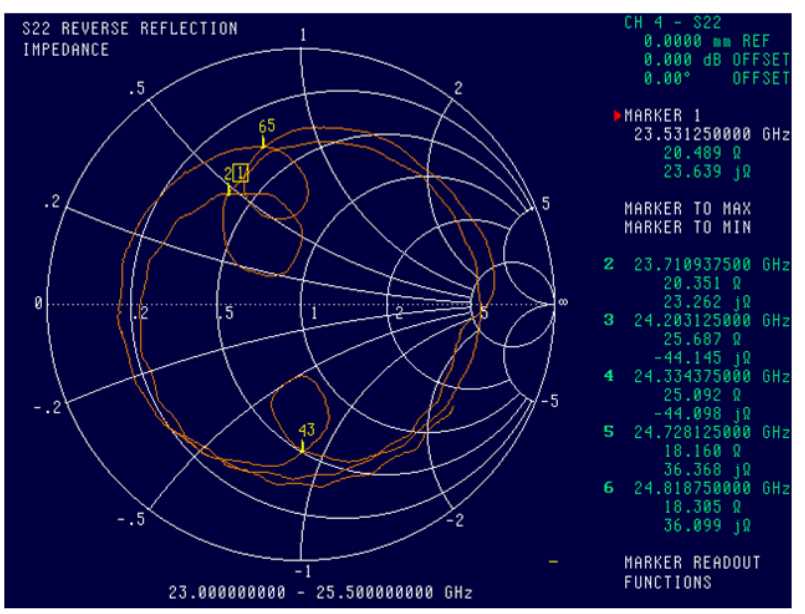

Fig. 5 The Smith chart for reflection $S_{22}$ of the closed cavity. Loops denoted by 21,43 and 65 are the $7 \pi / 10,8 \pi / 10$, and $9 \pi / 10$ axial modes, respectively.

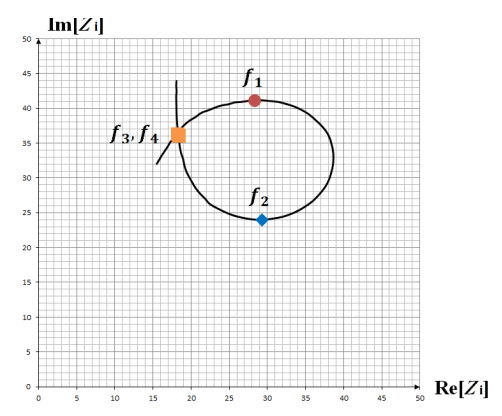

Fig. 6 The input-impedance $Z_{\mathrm{i}}$ locus in the vicinity of the $9 \pi / 10$ resonance for the closed cavity.

the detuned crossover point, at which the corresponding impedance is identical. By combining these four frequencies, $Q_{\Omega}$ is evaluated as

$$
Q_{\Omega}=\frac{f_{1}+f_{2}}{2\left|f_{1}-f_{2}\right|}|x|,
$$

where $|x|$ is a function of $f_{1}, f_{2}, f_{3}$, and $f_{4}$, given by Eq. (12) of Ref. [11]. This factor is called the modification factor of the critical-point bandwidth and is around one. By using the obtained $Q_{T}$ and $Q_{\Omega}, Q_{d}$ can be expressed as

$$
Q_{d}=\frac{1}{\frac{1}{Q_{T}}-\frac{1}{Q_{\Omega}}} .
$$

The relationship between the reflection coefficient at the structure end and $Q_{d}$ may be given as

$$
R=\sqrt{\frac{1-\alpha / Q_{d}}{1+\alpha / Q_{d}}}, \quad \text { and } \quad \alpha=\frac{\omega L}{v_{g}}
$$

where $v_{\mathrm{g}}$ is the group velocity of the spoof plasmon [5,9].

The quality factors and round-trip reflectance of the $9 \pi / 10$ axial mode for the closed cavity are presented in 
Table 1 Quality factor and round trip-reflectance $R$.

\begin{tabular}{|c|c|c|c|c|c|c|}
\hline & Axial & \multirow{2}{*}{$\begin{array}{c}\mathrm{f} \\
\text { Mode }\end{array}$} & \multicolumn{3}{|c|}{ Quality Factor } & $R$ \\
\cline { 4 - 6 } & {$[\mathrm{GHz}]$} & $Q_{\mathrm{T}}$ & $Q_{\Omega}$ & $Q_{\mathrm{d} \cdot}$ & {$[\%$} \\
\hline $\begin{array}{c}\text { Closed } \\
\text { Cavity }\end{array}$ & $9 \pi / 10$ & 24.8 & 407 & 508 & 2046 & 94 \\
\hline $\begin{array}{c}\text { Open } \\
\text { Cavity }\end{array}$ & $\begin{array}{c}\pi(9+1 / 2) \\
/ 10\end{array}$ & 24.8 & 283 & 452 & 758 & 84 \\
\hline
\end{tabular}

Table 1. For the closed cavity, the round-trip reflectance $R$ becomes $94 \%$, which corresponds to $97 \%$ for each $R_{1}$ or $R_{2}$. For the open cavity, the $\pi(9+1 / 2) / 10$ mode is very close to the $9 \pi / 10$ mode and the round-trip reflectance of $84 \%$ is obtained. Since the set-up on the port 1 side does not change, $R_{1}$ may be the same as $97 \%$. Hence, the value of $R_{2}$ changes from $97 \%$ to $87 \%$ by removing the reflector. The end reflectance of the bounded surface wave is so strong that resonators can be formed without the reflector at the corrugation end. On the other hand, resonator formation by a hybrid wave requires artificial reflectors.

\section{Discussion and Conclusion}

The critical-points method is checked by applying it to a straight waveguide resonator for which equations of $Q_{\Omega}$ are derived theoretically [15]. We prepare the waveguide made of aluminum alloy A5056 with a radius of $7 \mathrm{~mm}$ and a length $L_{\mathrm{s}}$ of $90 \mathrm{~mm}$. The experimental $Q_{\Omega}$ is about 3000 for the lowest $\mathrm{TM}_{01}$ resonant mode. The theoretical value is obtained as 6300 with the A5056's conductivity of 1.56 $\times 10^{7} \mathrm{~S} / \mathrm{m}$. Agreement between the experimental and theoretical values is good when we use a surface roughness factor of around 2. This factor depends on the surface conditions such as fabrication and oxidation and cannot be determined theoretically. A roughness factor of around 2 may be reasonable considering that the same factor has been reported for X-band corrugated waveguides [9, 13].

In Table 1, values of $Q_{\Omega}$ are on the order of hundreds and much lower than those for cavities with a roughness factor of $2[9,10,13]$. Our corrugations are fabricated without any special treatment such as mirror-like finish and the surface imperfections may reduce $Q_{\Omega}$. The effects of $Q_{\Omega}$ on the performance of spoof plasmon based devices are still unclear and should be studied more definitely for future applications.

In conclusion, by employing the cavity resonance method with VNA, we examine the dispersion characteristics and end reflectance of spoof plasmons formed on the metal cylinder with rectangular corrugations. The dispersion curve can be recognized as two regions; region (I) near the upper cutoff frequency and region (II) away from the upper cutoff frequency. The bounded surface wave is formed in region (I) and its end reflectance is strong enough to form resonators without any artificial reflector. On the other hand, the hybrid surface wave exists in region (II) and is similar to the Sommerfeld wave. Its end reflectance is too weak to form resonators. In such cases, formation of an axial resonator requires artificial reflectors on both ends of corrugation. This paper presents a useful method for examining the spoof plasmon, and may be considerably useful for developing electromagnetic wave sources and waveguiding systems based on period structure.

\section{Acknowledgments}

This work was partially supported by NIFS collaboration research program No. NIFS16KLER058 and No. NIFS18KLER074.

[1] J.B. Pendry et al., Science 305, 847 (2004).

[2] C.R. Willams et al., Nature Photonics 2, 175 (2008).

[3] S.A. Maier et al., Phys. Rev. Lett. 97, 176805 (2006).

[4] K. Ogura et al., J. Plasma Fusion Res. SERIES 6, 703 (2004).

[5] S. Gong et al., J. Appl. Phys. 118, 123101 (2015).

[6] Min Thu San et al., IEEE Trans. Plasma Sci. 46, 530 (2018).

[7] B. Levush et al., IEEE Trans. Plasma Sci. 20, 263 (1992).

[8] B. Levush et al., Phys. Fluids B 4, 2293 (1992).

[9] W. Main et al., IEEE Trans. Plasma Sci. 22, 566 (1994).

[10] S. Kobayashi et al., IEEE Trans. Plasma Sci. 26, 947 (1998).

[11] E.Y. Sun et al., IEEE Trans. Microw. Theory Tech. 43, 1983 (1995).

[12] K. Ogura et al., Plasma Fusion Res. 7, 2406022 (2012).

[13] M.R. Amin et al., J. Phys. Soc. Jpn. 65, 627 (1996).

[14] Y. Takashima et al., J. Plasma Fusion Res. SERIES 8, 1512 (2009).

[15] J.D. Jackson, Classical Electrodynamics, 2nd ed. (New York, NY, USA: Wiley, 1975) pp.356-360. 